levels in all patients positively correlated with CRP $(r=0.271, p=0.015)$. Furthermore, Hsp90 concentrations were negatively associated with functional parameters of ILD: FVC $(r=-0.291, p=0.013)$, FEV1 $(r=-0.248, p=0.036)$, DLCO $(r=-0.290, p=0.012)$ and $\mathrm{SpO}_{2}(r=-0.317, p=0.038)$. When adjusted for CRP, these correlations still remained significant in multivariate analysis. Higher Hsp90 concentrations were associated with presence of synovitis [17.6 (15.4 - 24.0) vs. 12.2 (9.3 - 17.3), $p=0.039]$. In addition, only in patients with dcSSc, Hsp90 levels positively correlated with the mRSS $(r=0.437, p=0.006)$. In a prospective analysis of patients with progressive SSc-ILD treated with $6(n=21$ patients) or 12 ( $n=14$ patients) monthly i.v. pulses of cyclophosphamide (CPA, $10 \mathrm{mg} / \mathrm{kg}$ ) we did not observe any significant differences between the baseline sample (month 0) and blood drawn after 1, 6 and 12 months. However, baseline Hsp90 was able to predict long-term response after one year of CPA treatment (DLCO $r=-0.494, p=0.037$ ). Moreover, change in Hsp90 after one month of CPA treatment $\left(\mathrm{Hsp}_{90} \mathrm{mi-m0}_{\mathrm{m}}\right)$ was able to predict the short-term inflammatory response $\left(\mathrm{CRP}_{\mathrm{m3}-\mathrm{m} 0}, \mathrm{r}=-0.495, \mathrm{p}=0.019 ; \mathrm{ESR}_{\mathrm{m3}-\mathrm{m0}}, \mathrm{r}=-0.496, \mathrm{p}=0.031\right)$. Concentrations of extracellular Hsp90 were not significantly affected by other main clinical parameters of SSc.

Conclusion: We demonstrated higher plasma levels of Hsp90 in SSc patients compared to healthy controls. Concentrations of extracellular Hsp90 increase with higher inflammatory activity, with deteriorated lung functions in ILD and also with the extent and severity of the skin involvement in patients with diffuse cutaneous SSc. These data further highlight the role of Hsp90 as a significant regulator of fibroblast activation and tissue fibrosis in SSc. In addition, Hsp90 could become a predictor of treatment response.

References:

[1] Tomcik M et al., Ann Rheum Dis.2014;73(6):1215-22

Acknowledgments: Supported by AZV-16-33542A, MHCR 023728 and SVV -260373 .

Disclosure of Interests: Hana Štorkánová: None declared, Sabina Oreska: None declared, Maja Špiritović: None declared, Barbora Heřmánková: None declared, Kristyna Bubova: None declared, Martin Komarc: None declared, Karel Pavelka Consultant of: Abbvie, MSD, BMS, Egis, Roche, UCB, Medac, Pfizer, Biogen, Speakers bureau: Abbvie, MSD, BMS, Egis, Roche, UCB, Medac, Pfizer, Biogen, Jiří Vencovský: None declared, Jörg Distler Grant/research support from: Boehringer Ingelheim, Consultant of: Boehringer Ingelheim, Paid instructor for: Boehringer Ingelheim, Speakers bureau: Boehringer Ingelheim, Ladislav Šenolt: None declared, Radim Bečvář Consultant of: Actelion, Roche, Michal Tomcik: None declared

DOI: 10.1136/annrheumdis-2020-eular.2726

\section{FRI0263 \\ QUANTITATIVE MUSCLE ULTRASOUND IN PATIENTS WITH IDIOPATHIC INFLAMMATORY MYOPATHIES-A PRELIMINARY STUDY}

A. Tripoli ${ }^{1}$, S. Barsotti ${ }^{1}$, A. Delle Sedie ${ }^{2}$, G. Aringhieri ${ }^{3}$, S. Vitali ${ }^{4}$, R. Neri ${ }^{5}$, D. Caramella ${ }^{6}$, M. Mosca ${ }^{7} .{ }^{1}$ Rheumatology Unit, Clinical and Experimental Medicine University of Pisa, Pisa, Italy; ${ }^{2}$ Rheumatology Unit, Department of Clinical and Experimental Medicine, University of Pisa, Pisa, Italy; ${ }^{3}$ Radiology, Department of Translational Research on New Technologies in Medicine and Surgery, University of Pisa, Italy, Pisa, Italy; ${ }^{3}$ Radiology, Department of Translational Research on New Technologies in Medicine and Surgery, University of Pisa, Italy, Pisa, Italy; ${ }^{2}$ Rheumatology Unit, Department of Clinical and Experimental Medicine, University of Pisa, Pisa, Italy; ${ }^{3}$ Radiology, Department of Translational Research on New Technologies in Medicine and Surgery, University of Pisa, Italy, Pisa, Italy; ${ }^{2}$ Rheumatology Unit, Department of Clinical and Experimental Medicine, University of Pisa, Pisa, Italy

Background: Muscle involvement is the most frequent clinical feature in patients with idiopathic inflammatory myopathies (IIM). In addition to muscular enzymes, muscular magnetic resonance has been investigated for the assessment of disease activity, but it is limited by high costs and it is not readily available. Muscle ultrasound (MUS) has been recently proposed as a valuable tool for the diagnosis and activity assessment of muscle involvement in IIM patients.

Objectives: To define the role of MUS in the diagnosis and assessment of disease activity in IIM through quantitative analysis of MUS

Methods: This was a prospective study conducted from February 2019 to November 2019. 41 patients with IIM: 17 men and 24 women, median age 61.4 years, of which 20 were polymyositis (PM), 16 dermatomyositis (DM) and 5 inclusion body myositis (IBM) were included. 30 healthy subjects (HS), comparable in age and gender to patients, were recruited as controls. In every patient and control MUS of upper and lower extremities was performed (in total 10 muscles per side) and digital images were saved. Quantitative muscle echo intensity (QME) was calculated using an image processing program (ImageJ) to obtain the mean value of greyscale (mGS) for each muscle. For patients with IIM creatine phosphokinase
(CPK) levels were recorded, duration of disease (in months) was calculated and clinical evaluation tools for the assessment of disease activity were performed, such as manual muscle testing (MMT8), patient and physician visual analogue scales (pVAS, phVAS), health assessment questionnaire (HAQ) and myositis disease activity assessment tool (MDAAT).

Results: Patients had higher values of mGS across all muscles examined than controls $(p<0.001)$. Among patients QME showed a negative correlation with MMT8 $(\mathrm{p}<0.001,-0.641<\mathrm{r}<-0.412)$, but no correlation with CPK levels or duration of disease. A positive correlation was found between QME and HAQ $(\mathrm{p}<0.05 ; 0.320<\mathrm{r}<0.599)$, pVAS $(0.003<\mathrm{p}<0.046 ; 0.314<\mathrm{r}<0.455)$ and $p h V A S$ $(0.029<\mathrm{p}<0.002 ; 0.341<\mathrm{r}<0.471)$. No significant correlation was found between QME and MDAAT and no statistically significant differences of muscle echo intensity were observed between patients with IBM, DM and PM.

Conclusion: Quantitative analysis of MUS showed to be useful to differentiate IIM patients from healthy subjects, therefore it could be a helpful technique to screen patients with muscular symptoms in which perform additional investigations. In our study, the data collected did not allow to assess the disease activity of IIM patients and did not allow to distinguish between the 3 different subgroups of IIM patients, but further studies may help in the identification of different muscular patterns to guide the clinical suspect and the possible role of MUS in the follow-up of the patients.

Disclosure of Interests: Alessandra Tripoli: None declared, Simone Barsotti None declared, Andrea Delle Sedie Speakers bureau: MSD, Lilly, Novartis, Abbvie, Celgene, Giacomo Aringhieri: None declared, Saverio Vitali: None declared Rossella Neri: None declared, Davide Caramella: None declared, Marta Mosca None declared DOI: 10.1136/annrheumdis-2020-eular.5856

\begin{tabular}{ll}
\hline FRI0264 & EFFECTIVENESS, SAFETY AND PATTERNS OF USE \\
& OF RITUXIMAB IN SCLERODERMA, IN CLINICAL \\
& PRACTICE: 9 YEARS' EXPERIENCE IN A TERTIARY \\
& HOSPITAL
\end{tabular}

E. Vicente $^{1}$, J. Fernández ${ }^{2}$, I. Llorente ${ }^{1}$, L. Vega ${ }^{3}$, S. Castañeda ${ }^{1,4}$,

A. García-Vadillo ${ }^{1}$, I. González-Álvaro ${ }^{1}$, A. Humbría ${ }^{1}$, A. Ortiz ${ }^{1}$, E. Patiño ${ }^{1}$, E. Tomero Muriel ${ }^{1}$, R. Garcia de Vicuna ${ }^{1}{ }^{1}$ H.U. de La Princesa, IIS-Princesa, Rheumatology, Madrid, Spain; ${ }^{2}$ Undergraduate Student of Medicine, UAM, H.U. de La Princesa, IIS-Princesa, Rheumatology, Madrid, Spain; ${ }^{3}$ H.U. de la Princesa, IIS-Princesa, Unidad Apoyo Metodológico, Madrid, Spain; ${ }^{4}$ Cátedra UAM-Roche, EPID-Future, UAM, Madrid, Spain

Background: Systemic sclerosis (SSc) is a clinically complex and heterogeneous disease. Interstitial lung involvement (ILD) is the main cause of mortality, but progression of skin fibrosis has also been associated with pulmonary dysfunction and mortality. Recently, Rituximab (RTX) has been postulated as a promising therapeutic alternative to cyclophosphamide (CF) or mycophenolate (MFM), but long-term experience is scarce.

Objectives: Describe the effectiveness, safety and long-term use of RTX, in a series of cases with SSc.

Methods: Retrospective observational study of patients with SSc (EULAR/ACR 2013 criteria) treated with RTX in a university hospital from 2010 to 2019 . Sociodemographic data related to SSc and treatments were collected. The effectiveness of RTX was evaluated at 6-12 months and at the end of follow-up, by means of these main outcomes: Rodnan's modified cutaneous index (mRSS) for skin fibrosis; CK leves for myopathy, variation $>10 \%$ in forced vital capacity (FVC) and $>15 \%$ in lung diffusion capacity of carbon monoxide (DLCO) for ILD. Adverse events $(A E)$ were recorded. Statistical analysis performed with Stata v.14 and statistical significance set for $\mathrm{p} \leq 0.05$.

Results: 14 women with SSc (mean age $47 \pm 13$ years, mean evolution $6.2 \pm 4.5$ years) were treated with RTX for ILD $(n=9)$, skin involvement $(n=11)$ and or inflammatory myopathy $(n=3)$. The mean $\pm S D$ of follow-up was $3.36 \pm 2.17$ years. SSc type: diffuse cutaneous $35.71 \%$, limited cutaneous $21.44 \%$, overlap $35.71 \%$ and sine scleroderma $7.14 \%$. Type of antibodies: $50 \%$ anti-Scl- 70 , $14.3 \%$ anti-centromere, $21.4 \%$ anti-RNA polymerase III and $7.14 \%$ anti-Ku. ILD was classified as NINE in 8 patients and NIU in 1. The first cycle of RTX included 2 infusions of $1 \mathrm{~g}$ and was initiated a mean of $3.36 \pm 2.17$ years after diagnosis. The retreatments were initially fixed every 6 months and later on demand in 4 patients, and in the rest on demand from the beginning, according to duration of clinical response. A mean of $3.9 \pm 2.5$ cycles/patient (range: 1-11) were administered. $30 \%$ of patients had previously received CF and $21.5 \%$ MFM. RTX was administered in association with other DMARDs (MTX $64.29 \%$, hydroxychloroquine [HCQ] $35.71 \%$, MFM $57.14 \%$, others $14.28 \%)$, CF $(14.29 \%)$, intravenous immunoglobulins $(7,14 \%)$ and prednisone $(78.57 \%)$. In the final visit, the percentage use of DMARDs (50\% MTX, 50\% MFM and $28.57 \% \mathrm{HCQ}$ ) and prednisone $(62.5 \%$ patients, $30 \%$ doses) was reduced. mRSS improved significantly. Muscle weakness disappeared in $3 / 3$ with normal CK levels in $2 / 3$ 